\title{
Strain related infectivity of Neisseria gonorrhoeae for the guinea-pig subcutaneous chamber and the variability of the immune resistance in different breeds of guinea-pig
}

\author{
P. NOVOTNY, E. S. BROUGHTON, K. COWNLEY, M. HUGHES, AND \\ W. H. TURNER \\ From the Department of Bacteriology, Wellcome Research Laboratories, Beckenham, Kent
}

SUMMARY The ability of gonococci to infect the subcutaneous chamber in guinea-pigs is strictly strain related. This ability was usually present in prototrophic strains and auxotrophs requiring proline only which were obtained from patients with uncomplicated gonorrhoea, but it was invariably absent in Neisseria gonorrhoeae auxotypes requiring arginine, uracil, and hypoxanthine, or arginine only for growth. All the strains obtained from disseminated gonococcal infections (all dependent upon arginine, uracil, and hypoxanthine) were unable to infect the guinea-pig chamber. Hence, the high invasiveness of $N$. gonorrhoeae for man and its infectivity for guinea-pig chambers appear to be unrelated properties. Although guinea-pigs of the same origin (Dunkin-Hartley) were used throughout, the degree of immune resistance was found to differ between the lines supplied by various breeders - that is, after a standard immunisation schedule using whole cell gonococcal vaccines, the homologous immune resistance to challenge varied from weak or non-existent in some lines, to highly resistant in others.

\section{Introduction}

The guinea-pig subcutaneous chamber model as pioneered by Arko (1972, 1973), is gaining interest in gonococcal research. This is because the $T_{1}-T_{2}$ colonial forms of gonococci can infect the chambers more easily than types $T_{3}-T_{4}$ and are able to survive therein for longer periods of time, and because active (Arko, 1974) and passive (Arko, 1974; Scales and Kraus, 1974) immunisation can be used to protect against an infectious challenge. Using this technique, it has been possible to (i) demonstrate several immunotypes (Arko et al., 1976a), (ii) show that pilar antibodies do not confer immunity to infection (Turner and Novotny, 1976), and that experimental cross-protection is related to bactericidal antibodies (Wong et al., 1976). Of greater significance is the finding that the increased immune resistance observed after the artificial infection or vaccination of chimpanzees broadly corresponds

Address for reprints: P. Novotny, Department of Bacteriology, Wellcome Research Laboratories, Beckenham, Kent BR3 3BS

Received for publication 26 September 1977 with results obtained in experiments with guinea pigs (Arko and Wong, 1977), and it is obvious tha guinea-pigs are attractive for in vivo studies of gonococcal physiology (Veale et al., 1975; Arko et al., 1976b; Penn et al., 1976, 1977a,b; Novotny et al., 1977).

It is not known whether the guinea-pig model has a direct relationship with the immune state in man. There is a basic difference between the infectious process in man and in guinea-pigs. In the latter infectious units are not formed (Novotny $e t$ al., 1977), and immunologically the guinea-pig recognises gonococci in a different way (Miler et al., 1977). Therefore, only exhaustive comparative studies would be able to show how knowledge obtained from a guinea-pig model would apply to the physiology and immunity of the gonococcal infection in man.

The guinea-pig model has been used extensively in this laboratory, and two qualities have become apparent. The first is related to the genotype of the gonococcus; certain freshly isolated gonococcal strains are non-infective for the chamber. The 
second is related to the genotype of the guinea-pigstwo distinct lines were found, one of which, when immunised, showed a high immune resistance to infection, while the other was barely protected.

\section{Methods}

\section{CULTURE MEDIUM}

GC medium plates were prepared from GC medium base (Difco) enriched with glucose $22 \mu \mathrm{mol} / \mathrm{ml}$, L-glutamine 0.68 $\mu \mathrm{mol} / \mathrm{ml}, \mathrm{Fe}\left(\mathrm{NO}_{3}\right)_{3} 0.1 \mu \mathrm{mol} / \mathrm{ml}$, and cocarboxylase $4.35 \times 10^{-4} \mu \mathrm{mol} / \mathrm{ml}$, all added as filter-sterilised solutions. The cultures were incubated at $37^{\circ} \mathrm{C}$ in jars containing 5 to $10 \% \mathrm{CO}_{2}$.

\section{GONOCOCCAL STRAINS}

The same strains were used as in previous studies (Novotny et al., 1975a,b; Novotny and Turner, 1975; Turner and Novotny, 1976; Novotny et al., 1977), all being obtained from the urethra and/or cervix of patients with gonorrhoea. The growth from primary cultures on GC medium or GC medium with VCN inhibitor (Baltimore Biological Laboratories) was washed off using a protective medium (Turner and Novotny, 1976) and stored in the gas phase of a liquid nitrogen refrigerator (LD40, Union Carbide Limited) as the primary master culture (PMC). When the primary cultures were contaminated, the $T_{1}-T_{2}$ colonies of gonococci were subcultured once on GC plates and then washed off and stored as the primary subculture (PS). To obtain seeds from frozen suspensions, the technique described by Bullen (1975) was used; using the serrated sampling tool a small amount of the suspension could be scraped from the frozen block, thus avoiding any thawing of the master suspensions. The cultures were identified by colonial morphology, oxidase, and fermentation tests (Kellogg and Turner, 1973). The cultures mainly consisted of $T_{1}$ or $T_{2}$ colonial forms. Where possible, subculturing, of $T_{3}$ and $T_{4}$ forms was avoided.

\section{AU XOTYPE DETERMINATIONS}

These were made using the NEDA media and the technique described by Catlin (1973) and Carifo and Catlin (1973). As after the recommended purification procedure, Difco Noble Agar was still inhibitory to some strains when used in the NEDA medium, $0.1 \%$ mass/vol. of soluble starch (Analar, BDH) was incorporated into the agar base (Professor Catlin, personal recommendation). Although impurities in the agar are usually blamed for the inhibitory effect, we found that the collection of glass bi-distilled water in a polyethylene receiver, rendered the water unsuitable and that the inhibitory effect thus caused could not be removed by adding starch. Therefore, contact with any sort of plastic during medium preparation was avoided. Individual batches of auxotyping media were checked by the use of the reference strains used by Carifo and Catlin (1973)-namely, strains ODH2915, MHD340, MHD361, SS7764/45, MCH3317/70, and MHD446 -all of which were obtained from the National Collection of Type Cultures, Colindale, London NW9 5HT.

\section{GUINEA-PIGS}

Dunkin-Hartley outbred animals, $500-800 \mathrm{~g}$, were supplied by Charles River, UK Limited, Margate, Kent; Porcellus Animal Breeding Limited, Heathfield, Sussex; Olac, Bicester, Oxon; Redfern Animal Breeders Limited, Brenchley, Kent.

\section{IMPLANTATION OF THE CHAMBERS}

The technique used was similar to that described previously (Turner and Novotny, 1976) with the following modifications. Twenty-four hours before surgery, the abdominal area of the guinea-pigs was clipped, and the guinea-pigs were supplied with drinking water containing $200 \mathrm{mg} /$ litre of Terramycin soluble powder (Pfizer Limited, Sandwich); this represented about $20-50 \mu \mathrm{g}$ of oxytetracycline per gram of animal weight a day. The supply of antibiotic was discontinued two to three days after chamber implantation. Ten minutes before surgery, the animals were injected intraperitoneally with $0.8 \mathrm{ml} / \mathrm{kg}$ body weight of $1 \%$ sodium thiopentone (Intraval Sodium, May and Baker, Dagenham). In some of the guinea-pigs a short inhalation of ether anaesthetic vapour was necessary to deepen the narcosis. The skin was thoroughly disinfected with $5 \%$ tincture of iodine and the narcotised animals were fixed within a sterile Mediplast sterilising bag (No. A-10, supplied by Henly's Medical Supplies, London N8) in which, using a sterile pair of scissors, a window of about $5 \times 3 \mathrm{~cm}$ had been cut in the transparent side of the bag, just above the operating area. This produced a clean surgical site' which was shielded from the rest of the guinea-pig. Through this window, two paracentral ventral incisions, $2 \mathrm{~cm}$ long, were made and then, using artery forceps, two subcutaneous pockets were prepared, into each of which two chambers were implanted. The chambers were positioned wide apart under the skin, which was then sutured using surgical braided nylon (Finlayson's metric no. 5) and finally dressed using Nobecutane spray (BDH). In this way each guineapig received four isolated chambers. The chambers were prepared from $2 \mathrm{ml}$ polypropylene heat sealable ampoules (no. 508S, Sterilin Limited, Teddington, Middlesex), which had had the narrow 
necks cut off and the sides perforated with 12 holes (approximately $2 \times 5 \mathrm{~mm}$ ) using a grinding wheel. The total area of the holes (about one square centimetre) represented approximately one-tenth to one-twelfth of the total surface area of the ampoule. The chambers were sterilised in an autoclave (15 minutes at $120^{\circ} \mathrm{C}$ ). The guinea-pigs were operated on in groups of five-usually 10 groups a day. Using this aseptic surgical technique, none of the implanted chambers became infected. If these precautions were neglected, this usually led to a high rate of chamber infection followed by subsequent rejection by some of the guinea-pigs.

\section{INFECTION OF THE CHAMBERS}

For this, cells harvested from the first subculture on GC plates were used. The plates were inoculated directly from the frozen PMC or PS and after 18-20 hours' incubation, colonies were resuspended in phosphate buffered saline (PBS) $(0.123 \mathrm{~mol} / \mathrm{l}$ $\mathrm{NaCl}, 0.01 \mathrm{~mol} / 1 \mathrm{Na}_{2} \mathrm{HPO}_{4}, 0.0032 \mathrm{~mol} / 1 \mathrm{KH}_{2} \mathrm{PO}_{4}$; pH 7.2) so that the injected volume of $0.2 \mathrm{ml}$ contained approximately $10^{7}$ colony forming units (cfu). The densities were adjusted spectrophotometrically (Coleman Junior II, at $650 \mathrm{~nm}$ ). Although the spectrophotometer readings had been calibrated for a number of strains, the actual number of cfu contained in the inoculum was found to be strain dependent and varied greatly $\left(10^{7}\right.$ to $\left.10^{9}\right)$. The actual number of cfu was therefore determined by plate counts (Novotny et al., 1977) in each case. The gonococcal suspensions were injected within 30 minutes of preparation, during which time no decrease in viable count was observed.

In several cases, the guinea-pig chambers were injected directly with pus from human males. Pus from the urethra was aspirated using a $1 \mathrm{ml}$ syringe and immediately emulsified with an equal volume of VCN inhibitor, which had been diluted 1:50 in PBS. The mixtures, usually 0.1 to $0.2 \mathrm{ml}$ in volume, were injected into the chambers after being left for 30 minutes at room temperature. Three to five days later, samples of the chamber contents were inoculated on to GC plates. About one-fifth of these samples rendered pure cultures of gonococci which were stored frozen as above.

\section{CHALLENGE SUSPENSIONS}

These were prepared from the PMC or PS in the same way as the suspensions for the chamber infection, or from the culture obtained from the infected chambers. Three challenge schedules were used:

1. PMC (or PS) $\rightarrow$ GC plate $\rightarrow$ challenge, or

2. PMC (or PS) $\rightarrow$ GC plate $\rightarrow$ GP chamber $\rightarrow$ GC plate $\rightarrow$ challenge, or
3. PMC (or PS) $\rightarrow$ GC plate $\rightarrow$ GP chamber $\rightarrow$ GC plate $\rightarrow$ frozen suspension $\rightarrow$ GC plate $\rightarrow$ challenge. The suspensions were diluted and standardised as above. The challenge suspension was regarded as adequate if it was possible to establish gonococcal infection in all of the inoculated chambers of the non-immune guinea-pigs, the infection having to persist for at least 14 days.

\section{TEST FOR CHAMBER INFECTION}

A small sample of the chamber contents (about $0.1 \mathrm{ml}$ ) was aspirated using a $2 \mathrm{ml}$ syringe and needle through the iodinated skin covering the chamber. One drop of this liquid (about $50 \mu \mathrm{l}$ ) was spread over a GC plate. Since the maximum volume of chamber liquid obtainable by this method was about $0.5 \mathrm{ml}$, this count volume represented about one-tenth of the chamber liquid. Inoculated plates were incubated for 18 to 24 hours, and the chamber was regarded as being infected when gonococci could be identified in the cultures. Growth varied from 1-2 colonies to confluent gonococcal growth. Each chamber was sampled several times-usually on the third and seventh day after gonococcal inoculation, and later at appropriate intervals. The chamber was regarded as being non-infected when no gonococcal growth was obtained on three successive occasions.

\section{AGGLUTINATION TEST}

The agglutination antigen was prepared as recommended by Arko (Arko, 1974, and personal communication). The growth from the first culture of the infected guinea-pig chamber liquid on GC medium was washed off with PBS, $0.5 \% \mathrm{v} / \mathrm{v}$ of $37 \%$ formalin was added, and the cell suspension was incubated for four hours at room temperature. The formolised cells were centrifuged $(3000 \mathrm{~g}$ for 10 minutes), and resuspended in PBS to a density of approximately $1 \mathrm{mg}$ dry weight of cells per $\mathrm{ml}$; the antigen was used on the day of preparation. Under these conditions, most strains gave a smooth suspension which did not autoagglutinate, but which deteriorated if kept at $4^{\circ} \mathrm{C}$ overnight.

Serial twofold dilutions of sera were made in plastic trays (U-shaped wells, IS-MRC-96, Linbro) using Microtitrator loops. An equal volume (0.05 $\mathrm{ml}$ ) of the antigen was added to each well, the trays were sealed with self-adhesive membranes, and then shaken to mix the reaction mixtures. After four hours' incubation at $37^{\circ} \mathrm{C}$ the plates were read using a Nikon plate microscope and oblique illumination. Positive results were compared with the control well (with serum omitted) and when autoagglutination occurred the test was repeated the next day with fresh antigen. The gonococcal sera were tested only against their homologous antigen. 


\section{VACCINES}

Gonococci from the frozen PMC or PS after one or two subcultures on GC plates were suspended in PBS containing $0.5 \% \mathrm{v} / \mathrm{v} 37 \%$ neutral formalin solution, incubated overnight at $4^{\circ} \mathrm{C}$, sedimented by centrifugation $(3000 \mathrm{~g}$, for 10 minutes), and resuspended in PBS. One $\mathrm{ml}$ of the final vaccine contained the equivalent of $10^{8} \mathrm{cfu}, 1 \mathrm{mg}$ of $\mathrm{A} 1$ (added as Alhydrogel, Superflos, Kobenhagen) and 1:10000 Merthiolate. The Alhydrogel was added routinely not primarily as an adjuvant, but as a detoxifying agent, since without it some of the vaccines were dermonecrotic. The vaccines were standardised in the same way as the challenge culture preparations. The vaccines were used within one and four weeks of their preparation. After storage at $4^{\circ} \mathrm{C}$ for about a year, the vaccines showed no apparent loss of protective activity under the particular test conditions, although considerable lysis of the cells was seen to have occurred when checked by an optical microscope.

For vaccines prepared from gonococci grown in embryonated eggs (10 days, White Leghorn), a previously reported modification (Novotny et al., 1977) of the method of Gibbs and Roberts (1975) was used. The seed eggs were inoculated with gonococci harvested from the first to third subculture on GC plates, and the culture from this passage in eggs was used to inoculate the production batch of eggs. After five hours' incubation at $37^{\circ} \mathrm{C}$ on an orbital shaker (Kuhner, Basel, Switzerland, type RC5SP) the allantoic fluid was harvested, centrifuged for 10 minutes at $800 \mathrm{~g}$ to remove the embryonal cells, and the supernatant respun at $6000 \mathrm{~g}$ to recover the gonococci. These were resuspended in PBS to a density of about $10^{8} \mathrm{cfu}$ per $\mathrm{ml}$ and formolised $(0.5 \% \mathrm{v} / \mathrm{v}, 37 \%$ formalin) overnight at $4{ }^{\circ} \mathrm{C}$. The formolised cell suspension after centrifugal washing was standardised as above.

COMPLEMENT TITRE DETERMINATION

The method of Mayer was used (Kabat and Mayer, 1961).

\section{Results}

INFECTIVITY OF FRESH ISOLATES OF GONOCOCCI FOR SUBCUTANEOUS GUINEA-PIG CHAMBER S

In most of our experiments, the strains tested for their ability to infect guinea-pig chambers were used as a suspension of colonies from the first to third subculture on GC medium. This passage on artificial medium was not necessary, since in several cases it was possible, in the presence of $\mathrm{VCN}$ inhibitor, to infect the chamber directly with human pus.
Initially, it appeared that all of our strains were infective-that is, they were able to establish an infection of the chamber, which subsequently lasted for several weeks, from an injection of approximately $10^{7}$ cfu from $T_{1}-T_{2}$ colonial forms. Later, the infection subsided spontaneously. Using fresh isolates the primary infection of the chambers did not usually last longer than two or three weeks. However, if the infected chamber contents were then transferred to a second guinea-pig chamber, or if the chamber liquid was cultured on GC plates and the colonies obtained by this single passage on artificial medium were used as a chamber inoculum, in most cases the infection of the chambers then lasted longer. With some strains-for example, Pat 1, StG 18, StG 4, StG 38-the infection lasted for six months or more (Turner and Novotny, 1976). It appears, therefore, that the survival period of gonococci in the guinea-pig chamber depends largely on selective conditions, passaged gonococci being eliminated from the chamber less readily than fresh isolates.

Eventually, we tried to test for passive protection in guinea-pigs using serum obtained from convalescents with disseminated gonococcal infection; experiments had shown that the serum, when administered intramuscularly, rapidly penetrated the chamber contents (Figure). However, we found

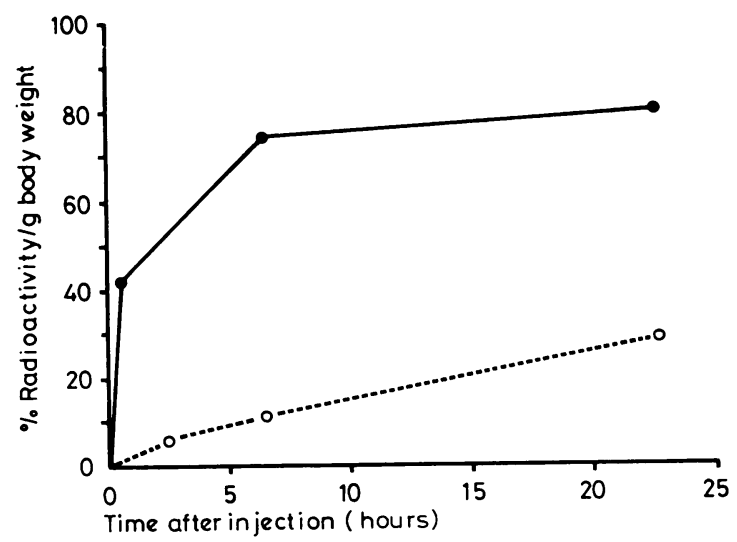

Figure Penetration of passively administered human antigonococcal IgG into subcutaneous chambers in guinea-pigs

Human antigonococcal IgG labelled with ${ }^{125}$ I (Novotny et al., 1977) was injected intramuscularly into seven guinea-pigs with non-infected chambers, samples were taken at appropriate intervals.

The results are expressed as the mean percentage of the total radioactivity injected per $\mathrm{g}$ of body weight. - Blood $\bigcirc---\ldots$ Chamber liquid 
that we were unable to infect the chambers using the strains obtained from these patients (Novotny et al., 1977). All of these strains behaved as typical DGI (disseminated gonococcal infection) strains (Holmes et al., 1976; Schoolnik et al., 1976)-that is, they were highly sensitive to penicillin, resistant to the bactericidal effect of human sera, and they required arginine, uracil, and hypoxanthine for growth. Despite intensive efforts to infect the chambers with these strains, testing for passive protection using human sera proved impossible, since using animal immune sera this test was found to be strain specific (Scales and Kraus, 1974, and unpublished observations). In the course of further experiments, other strains, which although originating from cases of uncomplicated gonorrhoea, were also found noninfective for the chamber. When auxotypes of all of our strains had been determined (Table 1) it became apparent, that none of the strains requiring arginine, uracil, and hypoxanthine for growthwhether from generalised infection or not-was infective. Another strain (StG 41) requiring arginine only, was also non-infective. On the other hand, with three exceptions, prototrophic strains or strains requiring proline only, obtained from patients with uncomplicated gonorrhoea, were able to establish infection when injected into the chamber. Two isolates from corresponding consorts, StG 102 and 104 (Novotny et al., 1975b), although auxotrophs requiring proline and arginine were infective. We suggest therefore, that the ability to infect the guinea-pig chamber is a specific characteristic of the wild strain, being absent in auxotrophic strains requiring arginine, uracil and hypoxanthine, but which, occasionally, may also be absent in some prototrophic strains. This inability to infect the chamber appears to be a stable feature, since isolates StG 90 and $174 \mathrm{C}$, when subcultured did not gain this property even after 10 passages on GC medium.
INDUCED IMMUNITY FOR GONOCOCCAL CHAMBER INFECTION

After preliminary experiments, a test system was developed which enabled us to perform routine determinations of the active immunity of guinea-pig chambers against gonococcal infection. In this test the guinea-pigs were injected intramuscularly or subcutaneously with two $1 \mathrm{ml}$ doses of a vaccine containing an equivalent of about $10^{8} \mathrm{cfu}$ per $\mathrm{ml}$ of gonococci in an aluminium gel. The doses were injected at an interval of between two and three weeks. The chambers, implanted one week before immunisation, were challenged with a suspension of the homologous strain containing approximately $10^{7} \mathrm{cfu}$. With most strains the size of the challenge did not appear to be critical, provided that the challenge dose fell between $10^{2}$ and $10^{8} \mathrm{cfu}$ (Table 2). Higher challenges were usually preferred since, with some fresh isolates, the control chambers occasionally failed to be infected by low challenge doses. Under these conditions, vaccines prepared from most of the infective strains listed in Table 1 induced solid immunity - that is, all the chambers were protected against the homologous challenge. This protection was independent of the nature of the challenge culture whether being in the form of a fresh isolate or an isolate obtained after one or several passages through the guinea-pig chambers. Vaccines prepared from some strains (StG 38, Pat 1) conferred protection even at a dilution of one-fifth of the neat vaccine dose, but were not usually protective at a dilution of $1: 25$. On the other hand, some fresh isolates conferred only feeble protection (for instance the vaccine prepared from strain StG 4 protected only six out of 17 chambers), while vaccines prepared from strains StG 18 and StG 76 were not able to protect any chamber. However, the protective potency of the whole cell vaccine could be increased by changing the conditions under which the vaccine cells were cultured. For instance

Table 1 Growth requirements and infectivity of $\mathrm{N}$. gonorrhoeae strains for subcutaneous guinea-pig chambers

\begin{tabular}{|c|c|c|c|c|c|c|}
\hline \multirow[b]{2}{*}{ Strain no. } & \multirow[b]{2}{*}{ Auxotype* } & \multicolumn{4}{|c|}{ Growth requirements* } & \multirow[b]{2}{*}{ Ability to infect guinea-pig chambers $\dagger$} \\
\hline & & Proline & Arginine & Hypoxanthin & Uracil & \\
\hline $\begin{array}{l}\text { StG } 2,4,6,18,19,25,38,76,79,86,87 \mathrm{U}, \\
93,94,95, \text { Pat } 1, \text { A4385 } \\
\text { StG } 48,90,174 \mathrm{C} \\
\text { StG } 41 \\
\text { StG } 102,104 \\
\text { StG } 127 \mathrm{C}, 127 \mathrm{U}, 128 \mathrm{C}, 128 \mathrm{U}, 176 \mathrm{C}, \\
176 \mathrm{U} \text { (= DGI strains) } \\
\text { StG } 45,77,80 \mathrm{C} \text { (DGI qualities not } \\
\text { tested) }\end{array}$ & $\begin{array}{l}1 \text { or } 2 \\
1 \\
3 \\
7\end{array}$ & $\begin{array}{l}- \text { or }+ \\
- \\
+ \\
+\end{array}$ & $\begin{array}{l}- \\
- \\
+ \\
+ \\
+\end{array}$ & $\begin{array}{l}- \\
- \\
- \\
+ \\
+\end{array}$ & $\begin{array}{l}- \\
- \\
- \\
+\end{array}$ & $\begin{array}{l}+ \\
- \\
+\end{array}$ \\
\hline
\end{tabular}

*Determined on NEDA medium according to Catlin (1973) and Carifo and Catlin (1973)

†The non-infective strains were tested on at least four chambers, most on 8 to 24 chambers and strains StG 90 and StG $174 \mathrm{C}$ on 65 and 84 chambers, respectively

†Disseminated gonococcal infection 
Table 2 Gonococcal protective test in Charles River guinea-pigs after immunisation with GC-plate grown vaccines

\begin{tabular}{|c|c|c|c|c|c|}
\hline \multirow{2}{*}{$\begin{array}{l}\text { Vaccine } \\
\text { strain }\end{array}$} & \multirow{2}{*}{$\begin{array}{l}\text { Homologous } \\
\text { challenge } \\
\text { dose (cfu) }\end{array}$} & \multirow{2}{*}{$\begin{array}{l}\text { Unvaccinated } \\
\text { controls }\end{array}$} & \multicolumn{3}{|c|}{ Vaccinated with no. of doses* } \\
\hline & & & 2 & 3 & 7 \\
\hline \multirow[t]{4}{*}{ StG 102} & $1.5 \times 10^{8}$ & $2 / 2 \dagger$ & $0 / 5$ & $2 / 6$ & $0 / 6$ \\
\hline & $3.0 \times 10^{6}$ & $2 / 2$ & $0 / 5$ & $0 / 6$ & $0 / 6$ \\
\hline & $6.0 \times 10^{4}$ & $3 / 3$ & $0 / 3$ & $0 / 6$ & $0 / 6$ \\
\hline & $1 \cdot 2 \times 10^{3}$ & $3 / 3$ & $0 / 5$ & $0 / 6$ & $0 / 6$ \\
\hline \multirow[t]{4}{*}{ StG 183} & $9.0 \times 10^{8}$ & $4 / 4$ & $0 / 4$ & $0 / 6$ & $0 / 6$ \\
\hline & $1.8 \times 10^{7}$ & $4 / 4$ & $0 / 4$ & $0 / 6$ & $1 / 6$ \\
\hline & $3.6 \times 10^{5}$ & $3 / 4$ & $0 / 5$ & $0 / 6$ & $0 / 6$ \\
\hline & $7 \cdot 2 \times 10^{3}$ & $3 / 3$ & $0 / 5$ & $0 / 6$ & $0 / 5$ \\
\hline
\end{tabular}

*The vaccine doses were applied intramuscularly at three-week intervals (when 2 or 3 doses were given) or one-week intervals in guinea-pigs receiving 7 doses. All guinea-pig chambers were challenged 10 days after the last vaccine injection

†The ratio represents the number of positive infections per number of chambers challenged

a vaccine prepared from strain StG 76 grown in the allantoic fluid of embryonated eggs, conferred protection after one vaccine dose (about half the chambers being protected) followed by solid immunity after two doses (six out of six chambers being protected). However, the same strain grown on GC plates did not confer protection even after two immunising doses of the vaccine, the cells having been subjected to a similar number of passages (Table 3). It is evident, therefore, that the production of the protective antigen(s) necessary for the development of an immune resistance in guinea-pig chambers may be largely dependent upon cultural conditions and that in some strains, this protective antigen may be present at a rather low level.

These tests were performed to investigate crossprotection between different strains. However, inconsistencies began to appear in our results, in that vaccines prepared from strains which had previously been found to be protective, failed to confer protection. It was originally supposed that cultural conditions were responsible. However, we later realised that these irreproducible results appeared when the Dunkin-Hartley guinea-pigs supplied by Charles River became temporarily unavailable, thus making it necessary to obtain guinea-pigs from other breeders. This can be seen by analysing the results of 1416 chambers, 686 of which were implanted into Charles River guineapigs, 342 into Olac or Redfern guinea-pigs, 300 into Porcellus guinea-pigs, and 88 into guinea-pigs of unknown origin. In the Olac and Redfern guineapigs, no homologous protection was conferred in $93 \%$ of the tests. In guinea-pigs supplied by Porcellus, the immune resistance was variable, some of the batches being well protected, while other batches failed to show protection with vaccines which had previously been shown to be protective. Only guinea-pigs supplied by Charles River rendered reproducible results, a situation which was confirmed when these guinea-pigs once again became available. As can be seen from Table 2, the protection is independent of both the number of immunising doses $(2,3$, or 7$)$ and the size of the challenge. On the other hand, in Olac and Redfern guinea-pigs the immune resistance where present is critically dependent upon the challenge dose (Table 3). Only those chambers which received relatively small challenge doses $\left(<10^{3} \mathrm{cfu}\right)$ appeared protected, whereas with higher challenge doses infection was established in many of the chambers. This remained the case even when the immunising dose was increased tenfold.

Table 3 Gonococcal protection test in Redfern and Charles River guinea-pigs after immunisation with two doses of vaccine (strain StG 76)

\begin{tabular}{lllll}
\hline $\begin{array}{l}\text { Vaccine } \\
\text { origin }\end{array}$ & $\begin{array}{l}\text { Homologous } \\
\text { challenge dose* } \\
(c f u)\end{array}$ & $\begin{array}{l}\text { Unvaccinated } \\
\text { controls }\end{array}$ & Vaccinated & Guinea-pigs \\
\hline Eggs & $6.0 \times 10^{7}$ & $5 / 5 \dagger$ & $6 / 10$ & $6 / 9$ \\
& $1.2 \times 10^{6}$ & $4 / 4$ & $5 / 10$ & $1 / 7$ \\
$2.4 \times 10^{4}$ & $4 / 5$ & $0 / 6$ & Redfern \\
Eggs & $4.8 \times 10^{2}$ & $5 / 5$ & $3 / 3$ & Charles River \\
GC-plate f & $2.0 \times 10^{7}$ & $8 / 8$ & & \\
\hline
\end{tabular}

*The guinea-pig chambers were challenged eight days after the final vaccine injection

tThe ratio represents the number of positive infections per number of chambers challenged 
A comparison of the agglutination titres of the sera between Charles River and Olac guinea-pigs showed that there was no difference in these two breeds (Table 4). Although there were more nonresponders among the Olac guinea-pigs, the general trend of these guinea-pigs to form high agglutination titres is apparent. However, despite this fact, the agglutination titre was found to be unrelated to the protective activity, as similarly reported by Arko et al. (1976b). Similarly, both groups of guinea-pigs showed high titres of antibodies reacting with free endotoxin particles and ring structures, both derived from the outer cell membrane, with particles derived from mesosome-like bodies and with pili, as determined by immune electron microscopy (Novotny and Turner, 1975; Novotny et al., 1977). Titres of antipilar antibodies were especially high, often being detectable at dilutions of 1:10000 or more (unpublished observations). Both groups of guinea-pigs were similar in their complement titre which was between $180-240 \mathrm{C}^{\prime} \mathrm{H}_{50}$ units per $\mathrm{ml}$.

\section{Discussion}

Some gonococcal strains are fastidious in their growth requirements, and strains obtained from patients with generalised infection usually belong to this category. This has been described in Seattle (Knapp and Holmes, 1975; Holmes et al., 1976; Schoolnik, 1976), and has led to the definition of a distinctive group of gonococci-the DGI strains-which are characterised by (1) their increased sensitivity to inhibitory factors in the culture media, (2) their low antibiotic resistance (especially to penicillin), (3) their resistance to the complement dependent bactericidal action of normal human sera, (4) their resistance or relative low sensitivity to the cidal effect of acute or convalescent sera, and, in most reported cases, (5) by their belonging to an auxotype requiring arginine, uracil, and hypoxanthine for growth. Although this relationship between the ability to produce the disseminated gonococcal infection and the markers mentioned is not absolute, there are a few exceptions. The determination of these properties appears to be important, since most of the highly invasive strains, and, probably, strains responsible for asymptomatic infections (Holmes et al., 1976), belong to this category. Hence an important laboratory criterion has been proposed for distinguishing this most virulent (invasive) and, from an epidemiological point of view, very important group of gonococci.

Although strains of gonococci which are noninfective for guinea-pig chambers (Arko and Wong, 1977) or for chimpanzees (Arko et al., 1974) are known, our study has shown that all of our auxotypes which require arginine, uracil and hypoxanthine for growth, including the DGI strains and a strain requiring arginine only were unable to infect guinea-pig chambers, whereas most prototrophic strains obtained from patients with uncomplicated gonorrhoea were infective. This property, therefore, could be added as another marker to differentiate between gonococcal strains. However, it also clearly shows that under laboratory conditions there is no direct relationship between the high virulence of gonococci for man and their infectivity for guinea-pig chambers. On the contrary, a strain which may be non-infective for the guineapig chamber, may have shown marked invasiveness for man. From this, it follows that the artificial increase in virulence of gonococcal isolates for the guinea-pig chamber (Arko et al., 1976b; Penn et al., 1977a), although interesting from the point of view of experimental infection, may not be directly comparable with the natural condition in man.

The current data indicate that non-infectivity for the guinea-pig chamber cannot yet be regarded as an irrefutable indicator of DGI properties, since some prototrophic strains were also found to be non-infective. Although on rare occasions, prototrophic strains have been obtained from disseminated gonococcal infection (Knapp and Holmes, 1975), as yet, no serious attempt has been made to distinguish them from the usual gonococcal strains using laboratory criteria. It also appears that although non-infectivity for the guinea-pig chamber is very often accompanied by specific growth requirements, these properties may occur independently. It should be possible to determine their relationship using genetic analysis.

Table 4 Agglutination titres of immunised guinea-pig sera against the homologous gonococcal strain. Serum samples taken 10-14 days after challenge

\begin{tabular}{|c|c|c|c|c|c|}
\hline \multirow{2}{*}{$\begin{array}{l}\text { Suppliers of } \\
\text { Dunkin-Hartley } \\
\text { guinea-pigs }\end{array}$} & \multirow{2}{*}{$\begin{array}{l}\text { Total no. of } \\
\text { guinea-pigs }\end{array}$} & \multicolumn{4}{|c|}{ Reciprocal of agglutination titres } \\
\hline & & 0 & $2-8$ & $16-24$ & $>128$ \\
\hline $\begin{array}{l}\text { Charles River } \\
\text { Olac }\end{array}$ & $\begin{array}{l}54 \\
66\end{array}$ & $\begin{array}{l}2 * \\
9\end{array}$ & $\begin{array}{r}20 \\
8\end{array}$ & $\begin{array}{l}19 \\
17\end{array}$ & $\begin{array}{l}13 \\
32\end{array}$ \\
\hline
\end{tabular}

*No. of guinea-pigs per titre group 
The finding that the properties of gonococcal strains can be determined experimentally in vivo has broadened the usefulness of the guinea-pig infectious model. On the other hand, however, its use in studies of immunological phenomena appears to be limited to the nutritionally less demanding strains - such as, those strains prevalent in uncomplicated symptomatic gonorrhoea. Despite this limitation, it would appear foolish to abandon this model in studies concerning the immunological and pathophysiological phenomena of these gonococci. Although the model is based upon the infection of an artificial cavity lined with fibrous tissue and not by mucosal epithelium, a characteristic inflammatory reaction develops (Chandler et al., 1976; Novotny et al., 1977). From the onset of the gonococcal infection, the cellular reaction has proliferative qualities which anatomically determine the chronic character of the infection (Novotny et al., 1977). It is this chronicity which bears the closest resemblance to the untreated disease in man (Chandler et al., 1976).

We are unable to explain why some breeds of guinea-pigs develop a high immune resistance after immunisation, whereas in other breeds the immune resistance is either weak or completely absent, thus rendering the size of the challenge critical. However, even with very low challenges (50 cfu), Penn et al. (1977b) were unable to confer protection in Olacbred guinea-pigs which had been immunised with five doses of gonococcal vaccines. On the other hand, Olac guinea-pigs are used in the Quality Control Department in these laboratories, and have been found suitable for the routine testing of several toxoids and whole cell vaccines and are, in these respects, comparable with guinea-pigs supplied by other breeders (Mr P. Knight, personal communication). It appears, therefore, that the differences observed in these experiments with gonococci are a direct consequence of the chamber model itself rather than of any general deficiency in the guinea-pigs. It is therefore apparent that some form of standardisation in the use of the chamber model is necessary if the results from different laboratories are to be compared. However, it is questionable whether any time devoted to an investigation into the immune resistance of the various lines of guinea-pigs would be relevant to our understanding of the disease in man.

We are grateful to Miss C. Briselden, Miss F. Smith, and Mrs B. Spacey for excellent technical assistance, and are deeply indebted to Professor B. W. Catlin (The Medical College of Wisconsin) and Dr R. J. Arko (CDC, Atlanta) for their advice on agar detoxification and gonococcal agglutination.
References

Arko, R. J. (1972). Neisseria gonorrhoeae: Experimental infection of laboratory animals. Science, 177, 1200-1201.

Arko, R. J. (1973). Implantation and use of a subcutaneous culture chamber in laboratory animals. Laboratory Animal Science, 23, 105-106.

Arko, R. J. (1974). An immunological model in laboratory animals for the study of Neisseria gonorrhoeae. Journal of Infectious Diseases, 129, $451-452$.

Arko, R. J., Bullard, J. C., and Duncan, W. P. (1976b). Effects of laboratory maintenance on the nature of surface reactive antigens of Neisseria gonorrhoeae. British Journal of Venereal Diseases, 52, 316-325.

Arko, R. J., Kraus, S. J., Brown, W. J., Buchanan, T. M., and Kuhn, U. S. G. (1974). Neisseria gonorrhoeae: Effect of systematic immunization on resistance of chimpanzees to urethral infection. Journal of Infectious Diseases, 130, 160-164.

Arko, R. J., and Wong, K. H. (1977). Comparative physical and immunological aspects of the chimpanzee and guinea-pig subcutaneous chamber models of Neisseria gonorrhoeae infection. British Journal of Venereal Diseases, 53, 101-105.

Arko, R. J., Wong, K. H., Bullard, J. C., and Logan, L. C. (1976a). Immunological and serological diversity of Neisseria gonorrhoeae: Immunotyping of gonococci by cross-protection in guinea-pig subcutaneous chambers. Infection and Immunity, 14, 1293-1296.

Bullen, J. J. (1975). A new technique for recovering bacteria stored in liquid nitrogen. Journal of General Microbiology, 89, 205-207.

Carifo, K., and Catlin, B. W. (1973). Neisseria gonorrhoeae auxotyping: Differentiation of clinical isolates based on growth responses on chemically defined media. Applied Microbiology, 26, 223-230.

Catlin, B. W. (1973). Nutritional profiles of Neisseria gonorrhoeae, Neisseria meningitidis, and Neisseria lactamica in chemically defined media and the use of growth requirements for gonococcal typing. Journal of Infectious Diseases, 128, 178-194.

Chandler, F. W., Kraus, S. J., and Watts, J. C. (1976). Pathological features of experimental gonococcal infection in mice and guineapigs. Infection and Immunity, 13, 909-914.

Gibbs, D. L., and Roberts, R. B. (1975). The interaction in vitro between human polymorphonuclear leucocytes and Neisseria gonorrhoeae cultivated in the chick embryo. Journal of Experimental Medicine, 141, 155-171.

Holmes, K. K., Eschenbach, D. A., and Knapp, J. (1976). Clinical epidemiology and change in clinical manifestations of gonococcal infection. In Sexually Transmitted Diseases, pp. 74-83. Edited by R. D. Catterall and C. S. Nicol. Academic Press: London.

Kabat, E. A., and Mayer, M. M. (1961). Complement and complement fixation. In Experimental Immunochemistry, second edition, pp. 133240. Thomas: Springfield.

Kellogg, D. S., Jr, and Turner, E. (1973). Rapid fermentation confirmation of Neisseria gonorrhoeae. Applied Microbiology, 25, 550-552.

Knapp, J. S., and Holmes, K. K. (1975). Disseminated gonococcal infections caused by Neisseria gonorrhoeae with unique nutritional requirements. Journal of Infectious Diseases, 132, 204-208.

Miler, J. J., Novotny, P., Walker, P. D., Harris, J. R. W., and MacLennan, I. P. B. (1977). Neisseria gonorrhoeae and ABO isohemagglutinins. Infection and Immunity, 15, 713-719.

Novotny, P., Short, J. A., Hughes, M., Miler, J. J., Syrett, C., Turner, W. H., Harris, J. R. W., and MacLennan, I. P. B. (1977). Studies on the mechanism of pathogenicity of Neisseria gonorrhoeae. Journal of Medical Microbiology, 10, 347-362.

Novotny, P., Short, J. A., and Walker, P. D. (1975a). An electron microscope study of naturally occurring and cultured cells of Neisseria gonorrhoeae. Journal of Medical Microbiology, 8, 413-427.

Novotny, P., and Turner, W. H. (1975). Immunological heterogeneity of pili of Neisseria gonorrhoeae. Journal of General Microbiology, 89, 87-92.

Novotny, P., Turner, W. H., Harris, J. R. W., and MacLennan, I. P. B. (1975b). Serology of Neisseria gonorrhoeae pili as a possible tool in the epidemiology of gonorrhoea. Proceedings of the Society for General Microbiology, 3, 58.

Penn, C. W., Parsons, N. J., Sen, D., Veale, D. R., and Smith, H. (1977b). Immunization of guinea-pigs with Neisseria gonorrhoeae: Strain specificity and mechanism of immunity. Journal of General Microbiology, 100, 159-166.

Penn, C. W., Sen, D., Veale, D. R., Parsons, N. J., Smith, H., and Witt, K. (1976). Morphological, biological and antigenic properties of Neisseria gonorrhoeae adapted to growth in guinea-pig subcutaneous chambers. Journal of General Microbiology, 97, 35-43. 
Penn, C. W., Veale, D. R., and Smith, H. (1977a). Selection from gonococci grown in vitro of a colony type with some virulence properties of organisms adapted in vivo. Journal of General Microbiology, 100, 147-158.

Scales, R. W., and Kraus, S. J. (1974). Development of passive transfer of immunity to gonococcal infection in guinea-pigs. Infection and Immunity, 10, 1040-1043.

Schoolnik, G. K., Buchanan, T. M., and Holmes, K. K. (1976) Gonococci causing disseminated gonococcal infection are resistant to the bactericidal action of normal human sera. Journal of Clinical Investigation, 58, 1163-1173.
Turner, W. H., and Novotny, P. (1976). The inability of Neisseria gonorrhoeae pili to confer immunity in subcutaneous guinea-pig chambers. Journal of General Microbiology, 92, 224-228.

Veale, D. R., Smith, H., Witt, K. A., and Marshall, R. B. (1975). Differential ability of colonial types of Neisseria gonorrhoeae to produce infection and an inflammatory response in subcutaneous perforated plastic chambers in guinea-pigs and rabbits. Journal of Medical Microbiology, 8, 325-335.

Wong, K. H., Arko, R. J., Logan, L. C., and Bullard, J. C. (1976). Immunological and serological diversity of Neisseria gonorrhoeae: Gonococcal serotypes and their relationship with immunotypes. Infection and Immunity, 14, 1297-1301. 